\title{
Skull base approaches to diseases involving the posterior fossa
}

\author{
Ali F. KRIShT, M.D. \\ Department of Neurosurgery, University of Arkansas for Medical Sciences, Little Rock, Arkansas
}

The complex anatomy of the posterior fossa as it relates to the skull base and the proximity of vital neurovascular elements provide a significant challenge when planning surgical approaches to lesions in this region. In the last 15 years major surgical advances were achieved in tackling posterior fossa lesions previously considered inoperable. These advances were made possible by a better understanding of skull base microsurgical and bone anatomy, which has enhanced exposures to difficult areas and improved safety.

This issue is focused on skull base approaches to diseases involving the posterior fossa and is privileged by outstanding contributors who are considered among the top experts in their field. We divided the articles into four different groups. The first includes an article reviewing the anatomical aspects used in guiding the location of suboccipital burr holes and craniotomies of the posterior fossa. This was followed by four articles focusing on approaches to vascular pathologies of the posterior fossa. The third section contains articles related to approaches to different tumor pathologies, covering the different transpetrosal, farlateral, transcondylar, and combined approaches. The factors determining the choice of the approach were addressed with examples of different pathological entities such as petroclival meningiomas, neuroenteric cysts of the cervicomedullary junction and tumors of endolymphatic sac. 
Authors 
Title

Neurosurg. Focus / Volume 19 / August, 2005 
Authors 